\title{
Acción tutorial y rendimiento académico de los estudiantes de Ciencias Contables - Administrativas y Economia de la Universidad Nacional del Altiplano
}

\author{
Tutorial action and academic performance of the students of \\ Accounting-Administrative Sciences and Economics of the National \\ University of the Altiplano
}

Erika Beatriz García Castro ${ }^{\text {a }}$

https://orcid.org/0000-0002-2141-2333

Andrés Vilca Mamani ${ }^{\text {b }}$

https://orcid.org/0000-0003-4183-8961

Universidad Nacional del Altiplano, Puno, Perú.

a egarcia@unap.edu.pe

Recibido: 12/04/2021

b avilca@unap.edu.pe

\section{Resumen}

La tutoría universitaria sigue un proceso de carácter formativo en el estudiante mediante la orientación, acompañamiento y asesoramiento en la dimensión académica y personal social, las dimensiones implican el aprendizaje, toma decisiones y la resolución de problemas que al final influyen en el rendimiento académico. Bajo este enfoque el objetivo de la investigación es evaluar el efecto de la tutoría universitaria en el rendimiento académico de los estudiantes de Ciencias Contables-Administrativas y Economía de la Universidad Nacional del Altiplano. La metodología de investigación es cuantitativa de tipo causal, utilizando el modelo de regresión lineal y los modelos probabilísticos Logit y Probit, la técnica de recolección de datos fue la encuesta y el instrumento utilizado es el cuestionario, con una muestra de 332 estudiantes. Los resultados demuestran que el programa de tutoría es una herramienta eficaz en la mejora del rendimiento académico de los estudiantes, a razón de que los estudiantes asistidos por la tutoría universitaria mejoran la nota promedio obtenida en 1.07 puntos y con una probabilidad de mejora del 19\%; asimismo, las sesiones de tutoría individual tuvieron mayor efecto que la tutoría grupal. Puesto que la acción tutorial orienta la enseñanza al logro de competencias académicas y genéricas.

Palabras clave: Rendimiento académico; tutoría grupal; tutoría individual y tutoría universitaria.

\begin{abstract}
University tutoring follows a process of a formative nature in the student through guidance, accompaniment and advice in the academic and personal social dimension, the dimensions imply learning, making decisions and solving problems that ultimately influence academic performance. Under this approach, the objective of the research is to evaluate the effect of university tutoring on the academic performance of students of AccountingAdministrative Sciences and Economics of the National University of the Altiplano. The research methodology is quantitative of the causal type, using the linear regression model and the probabilistic Logit and Probit models, the data collection technique was the survey and the instrument used is the questionnaire, with a sample of 332 students. The results show that the tutoring program is an effective tool in improving the academic performance of students, since the students assisted by the university tutoring improve the average grade obtained by 1.07 points and with a probability of improvement of $19 \%$; likewise, individual tutoring sessions had a greater effect than group tutoring. Since the tutorial action guides the teaching to the achievement of academic and generic competences.
\end{abstract}

Keywords: Academic performance; group tutoring; individual tutoring and university tutoring. 


\section{Introducción}

El sistema de tutoría universitaria es el proceso de acompañamiento durante la formación profesional. En este contexto, la tutoría toma el papel de acompañamiento, asesoramiento y maduración de competencias (García, 2008; Lobato y Guerra, 2016). Porque la función de la tutoría es la formación universitaria mediante la búsqueda del saber (Lázaro, 2008). Así como orientar y acompañar en forma individual o grupal (Benites, 2020).

Por medio de la tutoría el docente puede alcanzar una mejor comprensión de los problemas que enfrenta el alumno en su adaptación al ambiente universitario (Chong, 2017), y esto se debe a las horas que el profesor dedica al estudiante para resolver dudas, pero siempre con la comunicación e interacción (Obaya y Vargas, 2014). Porque es frecuente que el docente tutor realice motivación al estudio, de ahí depende las metas que persiguen en la actividad académica (Fernández, 2013). Además, los estudiantes con tutoría tienden a reportar niveles más altos de confianza en sí mismos (Zheng et al., 2020).

La acción tutorial es un proceso de acompañamiento al estudiante para que mejore su rendimiento académico, desarrollar hábitos de estudio, reflexión y conciencia social (Aguilar et al., 2017; Vera, 2017) y favorecer el desarrollo académico del alumno (Alonso et al., 2018; Guerrero et al., 2019). Al final genera una satisfacción y actitud positiva hacia la tutoría recibida en los estudiantes (Benítez, 2018; Caldera et al., 2015; Hernández et al., 2016).

Asimismo, las tutorías pretenden disminuir la deserción escolar, aumentar el rendimiento académico y orientar en cuestiones personales (Carrillo y Ruiz, 2017; Lopez et al., 2020; Quispe et al., 2017). A razón de que la tutoría permite un vínculo cercano con el estudiante, cumpliendo así la función formadora que permita al estudiante conocerse así mismo (Bausela et al., 2010).

Es así que la incorporación de programas de tutoría universitaria es una preocupación constante en las universidades para remediar el bajo nivel de aprovechamiento y rendimiento académico estudiantil, que al final repercute en la calidad profesional de los egresados (Posso, 2005), se puede ver que de cada cien estudiantes que ingresan a la universidad cerca de la mitad no logra culminar sus estudios ni obtener la graduación (Guzmán et al., 2009). El abandono de estudios y/o deserción de los alumnos se da por la carencia de orientación hacia el estudiante (Orlandoni et al., 2016, 2017).

De la misma forma los estudiantes de la de la Universidad Nacional del Altiplano de Puno (UNA, Puno) que egresan en promedio se titulan después de 2.7 años según el II Censo Universitario realizado en el año 2010, esto indica que claramente que la tutoría universitaria aún tiene deficiencias en su implementación, es por ende que la tutoría debe ayudar en la toma de decisiones y solución de problemas en los alumnos (Argüelles et al., 2013).

Los estudios demuestran, que los estudiantes con mejor rendimiento académico son los que cursan tutorías (García et al., 2012; Kim et al., 2021; Martínez et al., 2017; Orlandoni et al., 2016, 2017) con una eficacia en hábitos de trabajo y estudio (Fernández y Arco, 2011), y que la confianza del tutor se convierte en cambios positivos del tutorado que impacta en los aspectos académicos (Carrillo y Ruiz, 2017). Pero esta influencia positiva desaparece cuando las relaciones entre tutor y alumno no son tan buenas (Lopez et al., 2020).

Asimismo, la tutoría personal y la frecuencia de la tutoría se relaciona significativamente con el rendimiento académico (Cruz y Portocarrero, 2017). Pero se debe contar con tutores que no tengan mucha carga laboral (Guerra y Borrallo, 2018), por otro lado Vásquez et al. (2015) indica que la tutoría ayuda a los estudiantes a resolver las dudas académicas y mejorar habilidades de estudio. Pues la acción tutorial orienta la enseñanza docente y el aprendizaje de los alumnos hacia el logro de competencias académicas y profesionales (Cano, 2009).

Independientemente de la tutoría existen otros factores que determinan el rendimiento académico, como las habilidades interpersonales positivas, las redes sociales, habilidad de trabajar en equipo, los comportamientos sociales, la autoestima, el clima social en la familia y las habilidades de autorregulación emocional (Cruz et al., 2002; Jacob, 2002; Klug y Peralta, 2019; López, 2017; Mejía y Bautista, 2014; Mendoza, 2018; Nuñez y García, 2005; Oyarzún et al., 2012; Rytkönen et al., 2012; Samadzadeh et al., 2011; Santos, 2010).

Por otro lado, están las conductas alimentarias del estudiante (Zuniga et al., 2018), el número de 
asignaturas aprobadas en el primer año de carrera (Ibarra y Michalus, 2010) entre otros.

En la actualidad se observa que los alumnos presentan problemas de bajo rendimiento académico $\mathrm{y}$, por otro lado que, a pesar de que existe un servicio de tutoría universitaria no se recurre a él con la debida frecuencia (Cruz y Portocarrero, 2017).

Las Escuelas Profesionales de Ciencias ContablesAdministrativas y Economía de la UNA Puno, desarrollan la tutoría universitaria mediante sesiones individuales y grupales que conciernen en la dimensión académica y personal, pero a pesar de ello los alumnos presentan problemas de bajo rendimiento académico, reprobación de exámenes parciales y reprobación del curso, en algunos casos acompañado de ausentismo y deserción con mayor grado en los primeros semestres.

En los últimos años la tutoría universitaria se ha convertido en uno de los temas de mayor actualidad y relevancia en las tendencias y políticas educativas de la educación superior (López, 2017; Narro y Arredondo, 2013), por lo que la tutoría universitaria toma un papel importante en el proceso de enseñanza-aprendizaje, con el fin de mejorar el potencial de aprendizaje (Aguilar et al., 2017) y es un herramienta que permite ayudar a la formación del estudiante universitario en las dimensiones: académica, personal y profesional (Amor, 2012).

144 Es así que el estudio permitirá analizar el grado o nivel de cumplimiento de los objetivos del programa de tutoría en el rendimiento académico de los estudiantes. Asimismo, este indicador permitirá elaborar planes de mejora de la acción tutorial, y que coadyuven a garantizar el licenciamiento y acreditación de cada Escuela Profesional y propiciar el fortalecimiento de capacidades de los docentes tutores.

Con las consideraciones anteriores, se ha planteado como objetivo de investigación evaluar el efecto de la tutoría universitaria en el rendimiento académico de los estudiantes de Ciencias ContablesAdministrativas y Economía de la Universidad Nacional del Altiplano. Para ello se toma a los estudiantes, que son asistidos por el programa de tutoría, así como las sesiones individuales y grupales de tutoría sobre el rendimiento académico.

\section{Marco teórico}

\section{La orientación y tutoría en la educación superior}

Para Sanchiz "La orientación psicopedagógica es un proceso de ayuda continuo, inserto en la actividad educativa dirigido a todas las personas, que trata del asesoramiento académico, profesional y personal, que tiene el objetivo del desarrollo del sujeto mediante la capacitación para la autoorientación y para la participación activa en el entorno que vive" (2009, p. 23).

Según Bausela et al., (2010) y García (2008) señalan que la orientación en el ámbito universitario es un proceso de carácter formativo que se desarrolla a lo largo del ciclo vital de cada persona. La orientación es considerada como un proceso de ayuda en las personas en periodo de formación, como debe prevenir posibles dificultades y desarrollar el aspecto personal y académico del alumno (Amor, 2012). De ahí Ferrer define la tutoría universitaria como: "una actividad de carácter formativo que incide en el desarrollo integral de los estudiantes universitarios en su dimensión intelectual, académica, profesional y personal" (Ferrer, 2003, p. 72) y de esa forma el tutor como el "profesor que tutela la formación humana y científica de un estudiante y le acompaña en sus procesos de aprendizaje" (Lázaro, 2003); un tutor es quien representa a alguien, quien cuida, protege, vela o tiene bajo su responsabilidad a otro. En definitiva, un tutor es quien guía a alguien, dado que la tutoría, en cualquier caso, supone siempre tutela, guía, asistencia, apoyo y ayuda a una persona mediante la orientación y el asesoramiento (García, 2008).

Según Obaya y Vargas (2014) la tutoría no pretende cumplir un programa de estudio, más bien precisa las acciones a desarrollar con los estudiantes. El trabajo del tutor debe partir de un clima de confianza y respeto para involucrar a los alumnos en la participación de las sesiones de tutoría e involucrarlos de manera personal, el tutor no solo debe prestar atención en el aspecto académico también debe tomar en cuenta sus opiniones y problemáticas. El tutor debe favorecer el aprendizaje de los alumnos, ser guía, orientador $\mathrm{y}$ proveer las herramientas necesarias para que generen su propio conocimiento. Al respecto, García et al. (2012) comentan que una de las actividades prioritarias de la acción tutorial es el desarrollo de estrategias, como la organización del texto y programación de las actividades de estudio, 
contribuyen a facilitar la adaptación del estudiante al ambiente escolar para mejorar sus habilidades de estudio e incrementar su desempeño escolar.

La tutoría implica procesos de comunicación y de interacción de parte de los profesores; implica una atención personalizada a los estudiantes, en función del conocimiento de sus problemas, de sus necesidades y de sus intereses específicos. Es una intervención docente en el proceso educativo de carácter intencionado, que consiste en el acompañamiento cercano al estudiante, sistemático $\mathrm{y}$ permanente, para apoyarlo $\mathrm{y}$ facilitarle el proceso de construcción de aprendizajes de diverso tipo: cognitivos, afectivos, socioculturales y existenciales (Narro y Arredondo, 2013).

\section{Tutoría individual y grupal}

La tutoría individual es la orientación personalizada a un estudiante por parte del tutor designado durante la formación profesional universitaria, o cuando requieran atención individualizada (Vera, 2017). Para Sola y Moreno la tutoría individual es "aquella que se establece con el objetivo de orientar, guiar y ayudar al estudiante sobre cuestiones académicas, personales o profesionales" (2005, p. 137).

La tutoría grupal está relacionada con el proceso de orientación hacia los estudiantes además facilita la interacción con los agentes socializadores, se suscita el diálogo para que logren concientizar el conocimiento de sí mismo, sus necesidades e intereses, permitiendo la socialización, reflexión e integración entre los miembros del grupo (Mercado et al., 2011; Rojas et al., 2017).

\section{Rendimiento académico}

Tomando la conceptualización de Navarro (2003) el rendimiento académico es "un constructo susceptible de adoptar valores cuantitativos y cualitativos, a través de los cuales existe una aproximación a la evidencia y dimensión del perfil de habilidades, conocimientos, actitudes y valores desarrollados por el alumno en el proceso de enseñanza aprendizaje", asimismo es un indicador de desempeño escolar, cuyos rasgos se distinguen de cualquier resultado del proceso de enseñanza aprendizaje. Por otro lado, como criterio de operacionalizar el rendimiento académico es el uso de las calificaciones o notas (Cascón, 2000; Oyarzún et al., 2012).

\section{Materiales y métodos}

La metodología de investigación es cuantitativa y el diseño de investigación es causal o explicativa. La técnica utilizada para la recolección de datos para la variable explicativa (tutoría universitaria) fue la encuesta y para la variable dependiente son las notas del sistema académico. El instrumento utilizado en la investigación fue el cuestionario.

El instrumento utilizado fue validado a través del juicio de un experto. El resultado de la validez del instrumento fue aplicable. El instrumento utilizado fue sometido a la prueba de confiabilidad de Alpha de Cronbach: $\alpha=\left(\frac{k}{k-1}\right)\left(1-\frac{\sum_{i=1}^{k} S_{i}^{2}}{S_{t}^{2}}\right)$, donde k es el número de ítems (preguntas) del instrumento (cuestionario), $S_{i}^{2}$ : es la varianza de los ítems (desde 1...i) y $S_{t}^{2}$ es la varianza total del cuestionario (suma de las varianzas de los evaluadores); el análisis de consistencia varía entre 0 y 1 , los valores inferiores a 0.80 indica que los instrumentos utilizados son inconsistentes. Si el valor de $\alpha$ se sitúa entre 0.80 y 0.90 hablamos de fiabilidad; el valor máximo esperado de $\alpha$ es 0.90 , valores por encima de este se considera que existe redundancia o duplicación, porque califica los ítems (preguntas) con el mismo constructo de elemento, y deben ser eliminados (Da Silva et al., 2015, p. 132). El índice resultó 0.89 por ende el instrumento utilizado es confiable.

La población objeto de estudio son los estudiantes de ciencias Contables-Administrativas y Economía de la Universidad Nacional del Altiplano del semestre 2019-I. La población total asciende a 2463 estudiantes matriculados, de los cuales 864 corresponden a economía, 959 a contabilidad y 640 a administración. Se consideró el muestreo estratificado proporcional y aleatorio, obteniendo una muestra de 332 estudiantes, con una proporción de 116 estudiantes de la Escuela Profesional de Economía, 129 estudiantes de la Escuela profesional de Contabilidad y 86 estudiantes de la Escuela Profesional de Administración. Asimismo, del total de la muestra el 53\% (176) estudiantes pertenecen al programa de tutoría y el resto no tiene un tutor asignado.

Para evaluar el efecto de la tutoría en el rendimiento académico se utilizó el diseño estadístico de Mínimos Cuadrados Ordinarios y Modelos probabilísticos Logit y Probit. 


\section{Modelo de regresión lineal (Mínimos Cuadrados Ordinarios)}

$X_{i}=\alpha_{0}+\alpha_{1}$ Tutor $_{a}+\alpha_{2}$ se $_{\text {indiv }}+\alpha_{3}$ se $_{\text {grupal }}+\alpha_{4}$ tuto $_{\text {sen }}$

$+\alpha_{5}$ edad,$+\alpha_{6}$ trabaja $+\alpha_{7}$ inter $+\alpha_{8}$ comedor $+\varepsilon_{i}$

Donde, Xi: variable dependiente, es el promedio global de nota del alumno y está expresado de 0 a 20, $\alpha$ : captura el efecto de las variables explicativas en la variable dependiente, Tutor $_{a}$ : 1 si el alumno es asistido por un docente tutor y 0 caso contrario, $s e_{\text {indiv }}$ : número de sesiones de tutoría individual que realizó el docente, $s e_{\text {grupal }}$ : número de sesiones grupales a la que asistió el alumno, tuto ${ }_{\text {semes }}$ : número de semestres que fue asistido el alumno por un tutor, edad: edad del alumno, trabaja: 1 si el alumno trabaja y 0 caso contrario, inter: 1 si el alumno tiene internet en casa 0 caso contrario y comedor: 1 si es beneficiario del comedor universitario 0 caso contrario.

\section{Modelo probit}

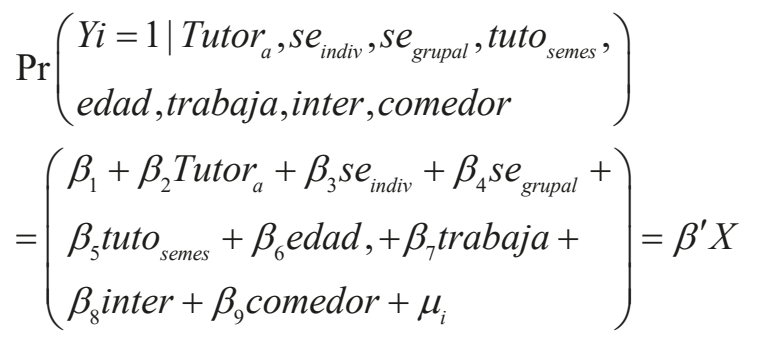

Donde, $\beta$ : Refleja el impacto que $\mathrm{x}$ tiene sobre la probabilidad y Yi: Probabilidad de mejora de la nota mayor al promedio estándar, 1 cuando el alumno tiene nota mayor al promedio estándar y 0 menor del promedio estándar.

$$
\operatorname{Prob}\left(Y=1 I X_{i}\right)=\frac{1}{\sqrt{2 \pi}} \int_{-\infty}^{=\beta^{\prime} X} e^{-\frac{t}{2}} d t=\Phi\left(\beta^{\prime} X_{i}\right)
$$

Donde $\Phi\left(\beta^{\prime} X_{i}\right)$, es la función de distribución normal estándar, que indica la probabilidad de mejora de la nota del alumno.

Efecto marginal para el modelo probit

$$
\frac{\partial E[y I x]}{\partial E}=\phi\left(\beta^{\prime} X_{i}\right) \beta
$$

El efecto marginal del modelo Probit permite calcular la incidencia de la tutoría en el rendimiento académico del alumno en porcentaje.

\section{Modelo Logit}

$\operatorname{Prob}(Y=1)=\frac{e^{\beta^{\prime} X_{i}}}{1+e^{\beta^{\prime} X_{i}}}=\wedge\left(\beta^{\prime} X_{i}\right)$

Donde $\wedge\left(\beta^{\prime} X_{i}\right)$ es la función de distribución logística.

$$
\frac{\partial E[y I x]}{\partial E}=\wedge\left(\beta^{\prime} X\right)\left[1-\wedge\left(\beta^{\prime} X\right)\right] \beta
$$

Una vez estimado los modelos Probit y Logit se podrá obtener el valor de la probabilidad de mejora de la nota cuando el alumno es asistido por un docente tutor a diferencia de otro alumno que no es asistido por un tutor.

\section{Resultados y discusión}

La estimación del modelo lineal no presenta problemas multicolinealidad ni correlación entre las variables regresoras. En cambio las pruebas de Breusch Pagan, indican la heterocedasticidad, para corregir esto se utiliza la robustez, las variables explicativas en la tabla 1 , columna 2 tienen un nivel de significancia al 5\%, excepto la variable comedor, y en la columna 1 las variables independientes edad y tutoría semestral no resultaron significativas, es por ello que esas variables se han omitido en el modelo.

Los resultados demuestran que el programa de tutoría en Ciencias Contables-Administrativas y Economía de la Universidad Nacional del Altiplano, tiene un efecto positivo sobre el rendimiento académico. En la tabla 1 columna 2 , indica cuando el estudiante es asistido por la tutoría universitaria difiere (aumenta) en 1.07 puntos sobre la nota promedio obtenida. El efecto positivo se debe a que la acción tutorial desarrolla estrategias y habilidades de aprendizaje en los alumnos que son asistidos por el programa de tutoría, complementando con el desarrollo personal y social.

El aprendizaje es un proceso interpersonal, de ahí el papel de la tutoría en la formación integral del estudiante mediante la orientación y el acompañamiento permanente. Además, la tutoría cumple el rol formativo en la dimensión académica, desarrollo personal y social.

En la figura 1, se representa la post estimación del modelo lineal gráficamente se puede corroborar 
un ligero ascenso de la nota del alumno cuando pertenece al programa de tutoría universitaria.

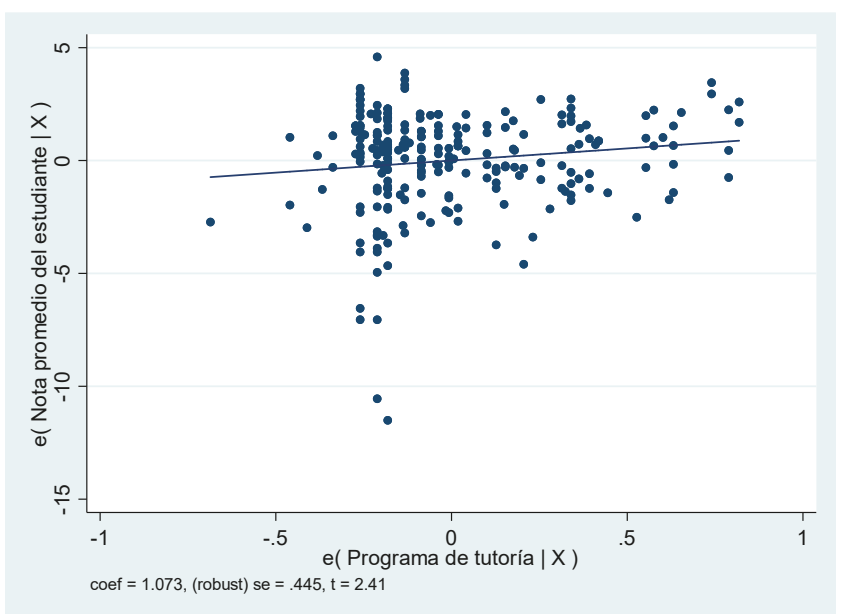

Figura 1. Post estimación de la tutoría universitaria y rendimiento académico.
De la misma forma, cuando el estudiante asiste a una sesión adicional de tutoría individual la nota promedio obtenida por el estudiante aumenta en 0.56 puntos (Tabla 1, columna 2). Esto se debe a que la tutoría individual provee las herramientas necesarias en el proceso de enseñanza-aprendizaje, mediante la orientación y acompañamiento permanente en desarrollo de estrategias de aprendizaje, sacar provecho al estudio, motivación al estudio, superar dificultades de estudio, aclarar dudas de aprendizaje, adaptación al estudio, informar sobre eventos académicos entre otros temas que necesita el estudiante.

Además, esto se debe a la orientación teórica del tutor, porque los estudiantes asimilan técnicas de aprendizaje, hábitos de estudio y resolución de dificultades personales, en vista de que la tutoría personal se enfoca al área académica, personal y familiar.

Tabla 1.

Resultados del modelo de regresión lineal del efecto de la tutoría sobre el rendimiento académico.

\begin{tabular}{|c|c|c|}
\hline \multirow[b]{2}{*}{ Variable } & (1) & (2) \\
\hline & \multicolumn{2}{|c|}{ Rendimiento académico Rendimiento académico } \\
\hline \multirow[t]{2}{*}{ Programa de tutoría } & $1.039 * *$ & $1.073 * *$ \\
\hline & $(2.22)$ & $(2.41)$ \\
\hline \multirow[t]{2}{*}{ Tutoría individual } & $0.541 * * *$ & $0.560 * * *$ \\
\hline & $(3.35)$ & $(3.64)$ \\
\hline \multirow{2}{*}{ Tutoría grupal } & $0.242 * *$ & $0.235 * *$ \\
\hline & $(2.13)$ & $(2.13)$ \\
\hline \multirow[t]{2}{*}{ Tutoría semestral } & 0.036 & \\
\hline & $(0.46)$ & \\
\hline \multirow[t]{2}{*}{ Edad del estudiante } & 0.027 & \\
\hline & $(0.38)$ & \\
\hline \multirow[t]{2}{*}{ Si trabaja el estudiante } & $-1.484 * * *$ & $-1.445 * * *$ \\
\hline & $(-4.41)$ & $(-4.55)$ \\
\hline \multirow[t]{2}{*}{ Conectividad } & $0.950 * * *$ & $0.941 * * *$ \\
\hline & $(3.15)$ & $(3.19)$ \\
\hline \multicolumn{2}{|c|}{ Beneficiario del comedor $0.752 *$} & $0.769 *$ \\
\hline & $(1.76)$ & $(1.83)$ \\
\hline \multirow[t]{2}{*}{ _cons } & $11.171 * * *$ & $11.770 * * *$ \\
\hline & $(6.86)$ & (31.66) \\
\hline $\mathrm{N}$ & 238 & 238 \\
\hline $\mathrm{r} 2$ & 0.327 & 0.326 \\
\hline $\mathrm{F}$ & 12.581 & 16.09 \\
\hline
\end{tabular}

En cuanto a la tutoría grupal se puede decir si el estudiante asiste a más de una sesión de tutoría grupal la nota promedio a obtener aumenta en 0.24 puntos, este efecto resultó inferior a la de tutoría individual, las sesiones de tutoría grupal son desarrollado por la unidad de tutoría por cada Escuela Profesional. Es así que las tutorías grupales e individuales no sólo favorecen la mejora de la nota sino también disminuyen el índice de reprobación, y así evitar que el alumno pase al riesgo académico 
con segunda o tercera matrícula. Si bien es cierto la tutoría grupal aborda temas de solidaridad, autoestima, responsabilidad, control emocional, estilos de aprendizaje entre otros temas.

Por otro lado en el modelo se incluyeron algunas variables secundarias como trabajo, si el alumno trabaja la nota disminuye en 1.45 puntos respecto al promedio; el resultado de conectividad o internet muestra si el alumno tiene internet en casa la nota promedio varía (aumenta) en 0.94 puntos respecto al promedio (Tabla 1, columna 3 ).

Asimismo, del apoyo social alimentario, si el alumno es beneficiario del comedor universitario la nota promedio obtenida aumenta en 0.77 puntos respecto a la nota promedio. El efecto positivo del servicio alimentario a estudiantes en el rendimiento académico, se debe a que la dirección de bienestar universitario promueve la alimentación saludable mediante la supervisión de personal capacitado en nutrición humana, es por esto que la nota del alumno beneficiario del servicio alimentario es superior a los de no beneficiarios.

Los resultados de los modelos probabilísticos Logit y Probit, también corroboran la mejora de nota cuando el estudiante forma parte del programa de tutoría universitaria. Aplicando la ecuación (5), la probabilidad de mejora del rendimiento académico según el modelo Logit es:

$$
\begin{gathered}
\operatorname{Prob}(Y=1)=\frac{e^{\left(-0.787+0.7895 \text { tutor }_{a}+0.463 \text { se }_{\text {indiv }}-1.092 \text { trabaja }+0.362 \text { inter }+0.613 \text { comedor }\right)}}{1+e^{\left(-0.787+0.7895 \text { tutor }_{a}+0.463 \text { e }_{\text {indiv }}-1.092 \text { trabaja }+0.362 \text { inter }+0.613 \text { comedor }\right)}} \\
\operatorname{Prob}(Y=1)=0.463
\end{gathered}
$$

Esto indica que en promedio la probabilidad de que específica en el modelo es del 46.3\%. el alumno mejore la nota promedio para la muestra

Tabla 2.

\begin{tabular}{|c|c|c|c|c|}
\hline Rendimiento académico & $\begin{array}{l}\text { (1) } \\
\text { Logit }\end{array}$ & $\begin{array}{l}(2) \\
d y / d x\end{array}$ & $\begin{array}{l}\text { (3) } \\
\text { Probit }\end{array}$ & $\begin{array}{l}\text { (3) } \\
d y / d x\end{array}$ \\
\hline $\operatorname{Prob}(\mathrm{Y}=1)$ & 0.463 & & 0.464 & \\
\hline Programa de tutoría & $\begin{array}{l}0.789 * * \\
(2.11)\end{array}$ & 0.192 & $\begin{array}{l}0.477 * * \\
(2.08)\end{array}$ & 0.186 \\
\hline Tutoría individual & $\begin{array}{l}0.463^{* * *} \\
(2.24)\end{array}$ & 0.115 & $\begin{array}{l}0.288^{* *} \\
(1.83)\end{array}$ & 0.114 \\
\hline Si trabaja el estudiante & $\begin{array}{l}-1.092 * * * \\
(-3.39)\end{array}$ & -0.259 & $\begin{array}{l}-0.666^{* * * *} \\
(-3.49)\end{array}$ & -0.255 \\
\hline Conectividad & $\begin{array}{l}0.362+ \\
(1.19)\end{array}$ & 0.089 & $\begin{array}{l}0.226+ \\
(1.25)\end{array}$ & 0.089 \\
\hline Beneficiario del comedor & $\begin{array}{l}0.613+ \\
(0.98)\end{array}$ & 0.151 & $\begin{array}{l}0.380+ \\
(1.05)\end{array}$ & 0.150 \\
\hline _cons & $\begin{array}{l}-0.787 * * \\
(-2.64)\end{array}$ & & $\begin{array}{l}-0.484 \\
(-2.68)\end{array}$ & \\
\hline $\mathrm{N}$ & 238 & & 238 & \\
\hline
\end{tabular}

Resultados del modelo probabilístico Logit y Probit.

Asimismo, aplicando la ecuación (6) en la sección anterior el efecto marginal para el modelo Logit es:

$$
\begin{gathered}
\frac{\partial E[y I x]}{\partial \text { tutor }_{a}}=0.463 *[1-0.463] * 0.789 \\
\frac{\partial E[y I x]}{\partial \text { tutor }_{a}}=0.192
\end{gathered}
$$

Esto afirma que si el estudiante es asistido por la tutoría universitaria la probabilidad de mejorar la nota promedio es del 19\% a diferencia de los estudiantes que no forman parte del programa de tutoría (Figura 2).

De la misma forma, si la sesión individual de tutoría aumenta en una unidad la probabilidad de que el alumno mejore su nota promedio es del 
$11.5 \%$ (Tabla 2, columna 2). Este efecto positivo de la tutoría personalizada también contrasta con los resultados del modelo lineal.

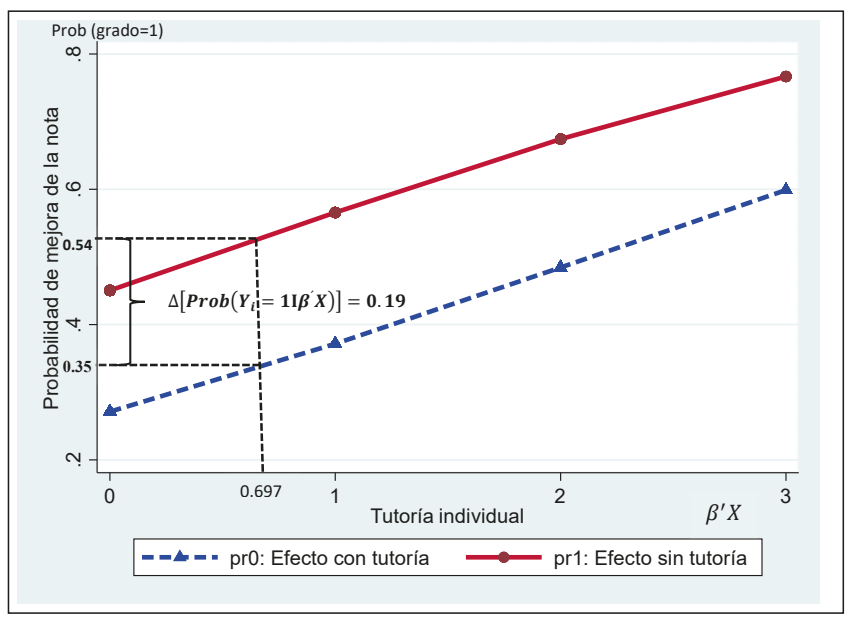

Figura 2. Probabilidad de mejora de la nota promedio como incidencia de la tutoría universitaria.

De la misma forma cuando el alumno trabaja el rendimiento académico disminuye en $25.9 \%$, el efecto es negativo al igual que en los resultados del modelo lineal. Las variables como; sesión grupal, comedor e internet no resultaron tan significativas en el modelo probabilístico.

\section{Discusión}

Los resultados demuestran, que la acción tutorial, así como la frecuencia de la tutoría individual y grupal, tuvo un efecto positivo en la mejora de nota promedio de los estudiantes. Los resultados concuerdan con el estudio de Cruz y Portocarrero (2017), quien comprueba una relación directa y significativa entre la tutoría universitaria y el rendimiento académico de los Alumnos de la Facultad de Ciencias Sociales de la Universidad Nacional Federico Villareal en el año 2015, esto demuestra que la tutoría universitaria sigue siendo una herramienta eficaz en la mejora de la nota del alumno, porque la tutoría según Alonso et al (2018) tiene una influencia positiva en el seguimiento de las metodologías activas.

El desempeño académico, que esta medido por el rendimiento académico, al final refleja en el logro de competencias, de ahí, Lobato y Guerra (2016) señala que la tutoria en la educacion superior cobra un relieve fundamental en la maduracion de competencias. Asimismo, la tutoria no solo influye en el rendimiento académico sino también en la formación integral, esto lo demuestra Martínez et al (2017) quien afirma que la tutoría influye en la formación integral de los estudiantes de medicina de la Universidad de Medellín en el año 2014.

El efecto positivo de la tutoría universitaria sobre el rendimiento académico, se debe a que la acción tutorial en forma conjunta desarrolla estrategias y habilidades de aprendizaje que han mejorado ligeramente el desempeño del alumno al aumentar las notas en las asignaturas. Tal como señala Vera (2017), donde menciona que la orientación de la tutoría a los estudiantes en problemas específicos de aprendizaje y desarrollo personal en las sesiones individuales de tutoría, el alumno mejora de forma significativa el rendimiento académico. Porque el tutor facilita las herramientas necesarias para que los estudiantes generen su propio conocimiento mediante un proceso de comunicación e interacción.

Del mismo modo, los resultados del presente estudio son coherentes con los resultados de Orlandoni et al (2017) quien señala, cuando los estudiantes cursan el programa de tutoría tienen 1.88 y 2.75 más posibilidades de aprobar el curso que un estudiante que no esté en el programa, realizado en la Facultad de Ciencias Exactas, Físicas y Naturales de la Universidad de Santander-Colombia en el año 2016. Pero el resultado solo hace referencia a la estimación logística de estudiantes de los primeros semestres y de un solo curso, mientras que la presente investigación toma la nota promedio de todos los cursos desde el segundo semestre al décimo semestre, utilizando la regresión lineal y modelos Logit y Probit; obteniendo así un efecto de la tutoría sobre el rendimiento académico en 1.07 puntos sobre el promedio con probabilidad de mejora de la nota del 19\%. Puesto que la acción tutorial no solo hace énfasis al proceso de enseñanza-aprendizaje, también hace énfasis al desarrollo personal, por ejemplo: la motivación al estudio, orientación profesional, responsabilidad, control emocional, trabajo en equipo etc. Todo esto ha complementado en incrementar la nota promedio del alumno.

Asimismo, la tutoría individual tuvo mayor efecto que la tutoría grupal en la mejora de la nota promedio obtenida por el estudiante, esto se debe a que la interacción entre estudiante y tutor es permanente en problemas de índole académico o desarrollo personal. El efecto es mayor porque los alumnos aprenden las técnicas de aprendizaje y hábitos de estudio del tutor y los estudiantes los ponen en la práctica (Carrillo y Ruiz, 2017), la 
acción favorable se debe también a que la tutoría es un espacio que se relaciona con la orientación teórica (Klug y Peralta, 2019). Porque la tutoría individualizada, se enfoca en el área: académica, personal y familiar, el aspecto académico de la tutoría personal aborda dificultades en la reprobación de exámenes parciales, trabajo en equipo, puntualidad, dificultades para exponer, dificultades para realizar y presentar trabajos. Porque la tutoría influye en la formación integral del estudiante (Martínez et al., 2017).

López et al (2020) afirma que el efecto positivo de la tutoría en el rendimiento académico es mayor cuando hay una buena relación entre tutor y alumno. En algunos casos no se ve la interacción permanente entre tutor y estudiante, esto se debe al temor del estudiante, o incluso a la cordialidad del tutor. Tal como señala Guerra y Borrallo (2018), las tutorías siempre en cuando son una estrategia eficaz para mejorar el rendimiento académico cuando se dé una serie de condiciones, como contar con buenos tutores, que no tengan sobrecarga y la comunicación debe ser de forma presencial y virtual. Así como brindarles un trato respetuoso, involucrarlos de manera personal considerando sus opiniones y problemáticas, y siempre con la confidencialidad de la información personal (Obaya y Vargas, 2014). Las escuelas profesionales carecen de ambientes de tutoría propiamente para los docentes tutores, muchas veces las sesiones se realizan en las aulas o pasadizos, esto juega un rol en contra de la confidencialidad, y del acercamiento permanente del estudiante hacia el tutor. Asimismo, la tutoría se debe brindar con un sistema de tutores en línea basado en la web, que ayuda a lograr mejor el aprendizaje (Muangprathub et al., 2020). Las Escuelas Profesionales aún no cuentan con la atención virtualizada, en la orientación y acompañamiento, existe un sistema web muy limitado, que solo sirve para hacer reportes básicos.

El efecto de la tutoría individual, sería mayor si se asigna un tutor durante todos los semestres, puesto que algunas veces los alumnos no todos los semestres son asistidos por la tutoría, además se prioriza a los estudiantes del primer al quinto semestre y a estudiantes en riesgo académico, o incluso se aplicará la tutoría por pares. Kim et al (2021), señala, que la tutoría por pares tiene múltiples beneficios, como reducir las tasas de reprobación de los cursos y aumentar las notas hasta en 5 puntos. Es claro que esta forma tutoría trae mejores resultados, sería muy bueno que el programa de tutoría incluya la tutoría por pares donde los alumnos de semestres superiores asistan a los alumnos de semestres inferiores.

La baja incidencia de la tutoría grupal sobre el rendimiento académico se debe a que las sesiones se desarrollan en grupos bastante grandes, muchas veces los ambientes no abastecen, por tanto no se garantiza la asistencia de la totalidad de los alumnos en las sesiones grupales. Además, la frecuencia de las sesiones es limitada, pero al igual que la tutoría individual hay un efecto positivo $y$ rescatable en la mejora de nota. Esto comprueba que las tutorías grupales ayudan a disminuir el índice de reprobación en los estudiantes (Argüelles et al., 2013). La tutoría grupal no sólo aborda temas en lo académico, también desarrolla temas de solidaridad, autoestima y responsabilidad. Porque la función de la tutoría es la formación universitaria mediante la búsqueda del saber (Lázaro, 2008).

La acción tutorial en las Escuelas Profesionales citadas, ha demostrado cumplir un rol integrador entre tutor y estudiante, e interacción del alumno con sus compañeros de aula, trabajo en grupo, adaptación al estudio, aclarar dudas de aprendizaje y sacar provecho al estudio. Porque el progreso académico, se relaciona positivamente con el apoyo de los compañeros, además el apoyo social y las habilidades de autorregulación (Rytkönen et al., 2012). De ahí que la acción tutorial ayuda a resolver problemas académicos como aprender hábitos de estudio del tutor, disminuir índices de reprobación de asignaturas y solucionar dificultades en el aprendizaje. Porque el aprendizaje, es un proceso interpersonal, es por ello que la tutoría busca acompañar durante la trayectoria universitaria (Vásquez et al., 2015).

\section{Conclusiones}

El programa de tutoría universitaria en Ciencias Contables-Administrativas y Economía de la Universidad Nacional del Altiplano, ha demostrado ser una herramienta eficaz en la mejora del rendimiento académico de los estudiantes, puesto que los estudiantes asistidos por la tutoría universitaria mejoran la nota promedio obtenida en 1.21 puntos y con una probabilidad de mejora del $15 \%$; el efecto positivo se debe a que el programa tutorial desarrolla habilidades y estrategias de aprendizaje, complementado con el desarrollo personal-social, que mejoran el desempeño del 
estudiante en aumentar las calificaciones, puesto que la tutoría cumple un rol de carácter formativo en la dimensión académico, personal y profesional.

Los resultados demuestran, que las sesiones de tutoría individual tuvieron mayor efecto positivo que la tutoría grupal en la mejora de las notas obtenidas de los estudiantes, por medio de la tutoría individual los profesores pueden alcanzar una mejor comprensión de los problemas que enfrenta el alumno en el proceso de enseñanza-aprendizaje, mediante la comunicación e interacción entre docente tutor y estudiante. Asimismo, la acción tutorial individual proporciona las herramientas necesarias para que los estudiantes generen su conocimiento; orienta a que los estudiantes asimilen técnicas de aprendizaje, hábitos de estudio, motivación al estudio, adaptación al estudio, sacar provecho del estudio, aclarar dudas de aprendizaje, sobre metodologías de aprendizaje, entre otros temas de orientación que requiere el estudiante.

Los resultados también demuestran que la tutoría grupal tiene un efecto positivo en el rendimiento académico, esto se debe a que las Escuelas Profesionales de Contabilidad, Administración y Economía desarrollan sesiones de tutoría grupal en temas de: motivación al estudio, responsabilidad, puntualidad, inteligencia emocional, trabajo en equipo, control de la impulsividad entre otros temas. Las variables secundarias como la conectividad (internet en casa) y cuando es beneficiario del apoyo social alimentario (comedor universitario), mejoran significativamente la nota promedio obtenida, en cambio cuando el estudiante trabaja el rendimiento académico disminuye.

\section{Conflicto de Intereses}

Los autores declaran no tener conflicto de intereses.

\section{Referencias bibliográficas}

Aguilar, W. E., Chávez, G. E., y Fuentes, M. (2017). Tutorías: Estudio exploratorio sobre la opinión de los estudiantes de Tronco Común de Ciencias de la Ingeniería. Formacion Universitaria, 10(3), 69-80. https://doi. org/10.4067/S0718-50062017000300008

Alonso, S. A., Rodríguez, A. M., y Cáceres, P. (2018). Análisis de la acción tutorial y su incidencia en el desarrollo integral del alumnado. El caso de la Universidad de Castilla La Mancha, España. Formación Universitaria, 11(3), 63-72. https:// doi.org/10.4067/S0718-50062018000300063

Amor, M. I. (Facultad de C. de la E. (2012). La orientación y la tutoría universitaria como elementos de calidad e innovación en la educación superior. Modelo de acción tutorial [Universidad de Cordova]. http://helvia.uco. es/xmlui/bitstream/handle/10396/7665/556. pdf? sequence $=1$

Argüelles, L. A., Quijano, R. A., y Fajardo, M. J. (2013). La práctica tutorial y su incidencia en el rendimiento académico. Caso FCAUAC Resumen Introducción. Revista Iberoamericana para la Investigación y el Desarrollo Educativo, 10, 1-11. 1-11.ride.org. $\mathrm{mx}$ > index.php > RIDESECUNDARIO , article $>$ download

Bausela, E., Pantoja, A., y Campoy, T. J. (2010). Planes de acción tutorial en la universidad. Revista de la Educación Superior, 34(153), 119-122. http://www.scielo.org.mx/pdf/resu/ v39n153/v39n153a8.pdf

Benites, R. M. (2020). El papel de la tutoría académica para elevar el rendimiento académico de los estudiantes universitarios. Revista Conrado, 16(77), 315-321.

Benítez, S. M. (2018). Tutoría Académica Universitaria: Percepción de estudiantes de la Facultad de Odontología. Universidad Nacional de Asunción. Revista Científica Estudios e Investigaciones, 7(1), 6-23. https:// doi.org/10.26885/rcei.7.1.6

Caldera, J. F., Carranza, M. D. R., Jiménez, A. A., y Pérez, I. (2015). Actitudes de los estudiantes universitarios ante la tutoría. Diseño de una escala de medición. Revista de la Educación Superior, 44(173), 103-124. https://doi. org/10.1016/j.resu.2015.04.004

Cano, R. (2009). Tutoría universitaria y aprendizaje por competencias: ¿cómo lograrlo? Revista Electrónica Interuniversitaria de Formación del Profesorado, 12(1), 181-204. https:// dialnet.unirioja.es/servlet/articulo?codigo $=2956810 \mathrm{yinfo}=$ resumenyidioma $=\mathrm{ENG}$

Carrillo, D., y Ruiz, M. I. (2017). La Tutoría : Una Herramienta Indispensable para el Estudiante Universitario. Salud y Administración, 4(10), 41-51. http://www.unsis.edu.mx/revista/doc/ vol4num10/5 La Tutoria.pdf

Cascón, I. V. (2000). Predictores del rendimiento académico en alumnos de primero y segundo de BUP. Unidad de Investigación de la 
Universidad de Salamanca. http://campus. usal.es/ inico/investigacion/jornadas/ jornada2/comun/c19.html

Chong, E. G. (2017). Factores que inciden en el rendimiento académico de los estudiantes de la Universidad Politécnica del Valle de Toluca. Revista Latinoamericana de Estudios Educativos, 47(1), 91-108.

Cruz, L., y Portocarrero, D. (2017). Tutoría universitaria y rendimiento académico de los alumnos de ciencias sociales de la Universidad Nacional [Universidad Nacional Federico Villarreal]. http:// repositorio.ucv.edu.pe/bitstream/handle/ UCV/5897/Cruz CL-Portocarrero CD.pdf?sequence $=1$ yisAllowed $=\mathrm{y}$

Cruz, M., Olvera, L., Domínguez, T., y Cortés, S. (2002). El papel de la inteligencia emocional en el rendimiento académico de estudiantes de ingeniería del INO. Revista Psicología y Salud, 12(2), 159-172.

Da Silva, F. C., Gonçalves, E., Valdivia, B. A., Bento, G. G., da Silva, T. L., Soleman, S. S., y da Silva, R. (2015). Estimadores de consistencia interna en las investigaciones en salud: el uso del coeficiente alfa. Rev Peru Med Exp Salud Publica, 32(1), 129-138. http:// www.scielo.org.pe/scielo.php?script $=$ sci arttextypid $=$ S1726-46342015000100019

Fernández, F., y Arco, J. (2011). Efectos de un programadeaccióntutorialentreuniversitarios. Infancia y Aprendizaje, 34(1), 109-122. https:// doi.org/10.1174/021037011794390120

Fernández, M. (2013). La motivación en la Tutoría. Una gran aliada. Revista de Claseshistoria, 396(2), 39-48. https://dialnet.unirioja.es/ servlet/articulo?codigo $=5174529$

Ferrer, J. (2003). La acción tutorial en la universidad. In F. Michavila, y J. García Delgado (Eds.). En La Tutoría y los nuevos modelos de aprendizaje en la Universidad (pp. 67-84). Madrid: Comunidad de Madrid. Dirección General de Universidades.

García, N. (2008). La función tutorial de la Universidad en el actual contexto de la Educación Superior. Revista Interuniversitaria de Formación de Profesorado, 22(1), 21-48. https://tutoria.unam.mx/sites/default/files/11functutorialsep.pdf

García, R. I., Cuevas, O., Vales, J. J., y Cruz, Ii. R. (2012). Impacto de la tutoría presencial y virtual en el desempeño académico de alumnos universitarios. Revista Iberoamericana de
Educación, 58(2), 1-11. https://doi.org/https:// doi.org/10.35362/rie5821447

Guerra, M. D., y Borrallo, Á. (2018). Tutoría y rendimiento académico desde la perspectiva de estudiantes y profesores de Ciencias de la Salud. Una revisión sistemática. Educación Médica, 19(5), 301-308. https://doi. org/10.1016/i.edumed.2017.03.019

Guerrero, J., Fuster, D., Gálvez, E., Ocaña, Y., y Aguinaga, D. (2019). Componentes predominantes de la acción tutorial en estudiantes universitarios. Propósitos y Representaciones, 7(2), 304-324. https://doi. org/10.20511/pyr2019.v7n2.300

Guzmán, C., Durán, D., y Franco, J. (2009). Deserción estudiantil en la educación superior colombiana. www.mineducacion. gov.co

Hernández, C. A., Jiménez, M., Guadarrama, E., y Rivera, Á. E. (2016). La Percepción de la Motivación y Satisfacción de la Tutoría Recibida en Estudios de Posgrado. Formación Universitaria, 9(2), 49-58. https://doi. org/10.4067/S0718-50062016000200006

Ibarra, M. del C., y Michalus, J. C. (2010). Análisis del rendimiento académico mediante un modelo logit. Ingeniería Industrial, 9(2), 47-56. file://E:/tes segunda didactica/resultados/ antecedentes/analisis del rendimiento con LOGIT.pdf

Jacob, B. A. (2002). Where the boys aren't: non-cognitive skills, returns to school and the gender gap in higher education. Economics of Education Review, 21, 589-598. http://citeseerx.ist.psu.edu/ viewdoc/download?doi=10.1.1.603.1097 yrep $=$ replytype $=$ pdf

Kim, S. C., Jillapali, R., y Boyd, S. (2021). Impacts of peer tutoring on academic performance of first-year baccalaureate nursing students: A quasi-experimental study. Nurse Education Today, 96(November 2020), 104658. https:// doi.org/10.1016/j.nedt.2020.104658

Klug, M. A., y Peralta, N. S. (2019). Tutorías universitarias. Percepciones de estudiantes y personal tutor sobre su uso y funcionamiento. Revista Electrónica Educare, 23(1), 319-341. https://doi.org/10.15359/ree.23-1.16

Lázaro, A. (2003). Competencias tutoriales en la universidad. In F. Michavila, y J. García Delgado (Eds.). En La tutoría y los nuevos modos de aprendizaje en la universidad (pp. 107-152). Madrid: Comunidad de Madrid. 
Dirección General de Universidades.

Lázaro, Á. J. (2008). Diferencias cualitativas entre experiencias tutoriales para opciones de aprendizaje universitario. Revista Interuniversitaria de Formación del Profesorado, 22(1), 109-137.

Lobato, C., y Guerra, N. (2016). La tutoría en la educación superior en Iberoamérica: Avances y desafíos. Educar, 52(2), 379-398. https://doi. org/10.5565/rev/educar.726

López, E. (2017). El concepto y las finalidades de la tutoría universitaria: una consulta a expertos. Revista Española de Orientación y Psicopedagogía, 28(2), 61-78.

Lopez, L. A., Marcenaro, O. D., y Molina, J. A. (2020). School tutoring and academic performance: A too close relationship? Studies in Educational Evaluation, 66, 100903. https:// doi.org/10.1016/j.stueduc.2020.100903

Martínez, L. M., Conejo, F., y Rodríguez, A. V. (2017). La acción tutorial como experiencia educativa para la formación integral de los estudiantes de Medicina. Actualidades Investigativas en Educación, 17(3), 1-23. https://doi.org/http://dx.doi.org/10.15517/aie. v17i3.30099

Mejía, R., y Bautista, A. (2014). El Hábito de las Redes Sociales en Estudiantes de Medicina. Bol Clin Hosp Infant Edo Son, 31(1), 19-25.

Mendoza, J. R. (2018). «Uso Excesivo de Redes Sociales de Internet y Rendimiento Académico en Estudiantes de Cuarto Año de la Carrera De Psicología UMSA». Educación Superior, 5(2), 58-69. http://www.scielo.org. bo/pdf/escepies/v5n2/v5n2 a08.pdf

Mercado, H., Palmerin, M., y Sesento, L. (2011). La tutoría grupal en la educación. Universidad de Malaga-Eumed.net. http://www.eumed. net $/ \mathrm{rev} / \mathrm{ced} / 31 / \mathrm{vcg} . h \mathrm{tml}$

Muangprathub, J., Boonjing, V., y Chamnongthai, K. (2020). Learning recommendation with formal concept analysis for intelligent tutoring system. Heliyon, 6, e05227. https:// doi.org/10.1016/j.heliyon.2020.e05227

Narro, J., y Arredondo, M. (2013). La tutoría un proceso fundamental en la formación de los estudiantes universitarios. Perfiles Educativos, 35(141), 132-151. http://www. scielo.org.mx/pdf/peredu/v35n141/v35n141a9. pdf

Navarro, R. E. (2003). El rendimiento académico: concepto, investigación y desarrollo. 1(2). http://www.ice.deusto.es/rinace/reice/volln2/ Edel.pdf
Nuñez, D. A., y García, C. R. (2005). Habilidades sociales, clima social. Periódicos Electrónicos en Psicologia, 11(11), 63-74.

Obaya, A., y Vargas, Y. M. (2014). La tutoría en la educación superior. Educación Química, 25(4), 478-487. https://doi.org/10.1016/S0187893X(14)70070-9

Orlandoni, G., Pérez, M., Aguilar, F., y Ramoni, J. (2017). ¿Puede un programa de tutorías mejorar el rendimiento académico de los estudiantes universitarios? Artículo de Investigación Científica y Tecnológica, 5(1), 4-16. https://doi.org/10.15649/2346075X.446

Orlandoni, G., Ramoni, J., Pérez, M., y Aguilar, F. (2016). Impacto del Programa de Tutorías en el Rendimiento Académico de Estudiantes Universitarios. Un Análisis de Diferencia en Diferencias (pp. 8-12).

Oyarzún, G., Estrada, C., Pino, E., y Oyarzún, M. (2012). Habilidades sociales y rendimiento académico: una mirada desde el género. Acta Colombiana de Psicología, 15(2), 21-28. http:// www.scielo.org.co/pdf/acp/v15n2/v15n2a03. pdf

Posso, A. (2005). Sobre el bajo aprovechamiento en el curso de Matemáticas I de la UTP. Scientia et Technica, 2(28), 169-174. https://doi.org/ http://dx.doi.org/10.22517/23447214.6853

Quispe, G. M., Ayaviri, D., y Arellano, E. (2017). Aplicación del Análisis Factorial en la medición de la calidad de la tutoría académica universitaria. Revista Espacios, 38(50), 16-42. https://www.revistaespacios.com/a17v38n50/ a17v38n50p16.pdf

Rojas, A. L., Estévez, M. A., y Domínguez, Y. (2017). Concepción de las tutorías, desde un enfoque inclusivo, en la carrera de Talento Infantil de la Universidad Metropolitana de Ecuador. Universidad y Sociedad, 9(2), 225231. http://rus.ucf.edu.cu/index.php/rus

Rytkönen, H., Parpala, A., Lindblom, S., Virtanen, V., y Postareff, L. (2012). Factors affecting bioscience students' academic achievement. Instructional Science, 40(2), 241-256. https:// doi.org/10.1007/s11251-011-9176-3

Samadzadeh, M., Abbasi, M., y Shahbazzadegan, B. (2011). Survey of Relationship between psychological hardiness, thinking styles and social skills with high school student's academic progress in Arak city. Procedia - Social and Behavioral Sciences, 28(2011), 286-292. https://doi.org/10.1016/j. sbspro.2011.11.055 
Sanchiz, L. (2009). Modelos de orientación e intervención psicopedagógica (Publicación). Colección Sapientia. www.sapientia.uji.es

Santos, M. (2010). Análisis de redes sociales y rendimiento académico: lecciones a partir del caso de los Estados Unidos. En Debates en sociología (Vol. 35).

Sola, T., y Moreno, A. (2005). La acción tutorial en el contexto del Espacio Europeo de Educación Superior. Educación y Educadores, 8, 123-143. https://www.redalyc.org/pdf/834/83400810. pdf

Vásquez, C. R., Espino, P., y Olaguez, J. E. (2015). Repercusiones de la tutoría académica en estudiantes de ingeniería. Revista Iberoamericana para la Investigación y el desarrollo Educativo, 5(10), 238-255.

Vera, M. del R. (2017). Efecto de un programa de tutoría en formación integral sobre el rendimiento académico de los estudiantes de tercer año de la Facultad de Ciencias de la Salud de la Universidad Técnica de Babahoyo. [Universidad Nacional Mayor de San Marcos]. https://bit.ly/346bw50

Zheng, X., Wang, C., Shen, Z., y Fang, X. (2020). Associations of private tutoring with Chinese students' academic achievement, emotional well-being, and parent-child relationship. Children and Youth Services Review, 112(18), 104934. https://doi.org/10.1016/j. childyouth.2020.104934

Zuniga, S., Pizarro, R., Escudero, M. E., y Honores, G. (2018). Rendimiento Académico Universitario y Conductas Alimentarias. Formación universitaria, 11(2), 57-64. https:// doi.org/10.4067/S0718-50062018000200057 